$16^{\text {th }}$ International Congress of Metrology, 06018 (2013)

DOI: $10.1051 /$ metrology/201306018

(c) Owned by the authors, published by EDP Sciences, 2013

\title{
Non destructive contactless precision measurement of flatness by moiré interferometry.
}

\author{
Said Meguellati ${ }^{1, a}$, and Aissa Manallah ${ }^{1}$ \\ ${ }^{1}$ Applied Optics Laboratory, Optics and Precision Mechanics Institute, University of Sétif 1- Algeria
}

\begin{abstract}
This optical scan method presented in this paper is used for precision measurement defects in flatness or absolute forms in comparison with a reference component form, of optical or mechanical components, surfaces. The principle of the method is to project the image of the source grating to palpate optically surface to be inspected, after reflection; the image of the source grating is printed by the object topography and is then projected onto the plane of reference grating for generate moiré fringe for defects detection. The optical device used allows a significant dimensional surface magnification of the surface inspected, which allows easy processing and reaches an exceptional precision of measurements. According to the measurement principle, the sensitivity for displacement measurement using moiré technique depends on the spatial frequency grating, for increase the detection resolution. This measurement technique can be used advantageously to measure the deformations generated by the production process or constraints on functional parts and the influence of these variations on the function.
\end{abstract}

\section{Introduction}

Moiré topography [1-7] is a technical field method in which the form of the object surface is measured by means of geometric interference produced by the superposition of two identical lines gratings. The technique has found many applications in various fields ranging from biomedical research to industrial and scientific applications. In many industrial measurement applications, the shape control by non-destructive and contact less measurement has become a desirable tool in control of quality and outline mapping. The optical system developed here, uses the technique of moiré [8-9] to highlight existing shape defects or deformation occurring in real time and continuously on reflective surfaces or reflective sufficiently. The control method presented in this context using an optical arrangement which allows use of gratings with high spatial frequency, which gives it the ability to reveal micro defects or deformities and measure them with high accuracy.

\section{Detection of flatness defects}

\subsection{Principle and operation of experimental setup}

The Laser beam HeNe $35 \mathrm{~mW}$ emitted by the source $\mathrm{S}$, and slightly expanded by the lenses system $\left(\mathrm{L}_{1}, \mathrm{~L} 2\right)$ depending on the inspected surface, and illuminates linear transmission grating $\mathrm{G}_{1}$; Figure 1. After crossing the grating $G_{1}$, the light beam is diffracted by a set of divergent rays that pass through the lenses system $\left(\mathrm{L}_{3}\right.$, $\mathrm{L}_{4}$ ) which realised the double Fourier transformation for to project the grating image $G_{1}$ on the plan mirror $M$ of high quality surface. The grating $\mathrm{G}_{1}$ image is reflected by the mirror $M$ on the grating $G_{2}$ plan, where it is optically superimposed on the linear transmission grating $\mathrm{G}_{2}$. Gratings $G_{1}$ and $G_{2}$ are identical and have same spatial frequency. In this case, the superposition of the grating image $G_{1}$ on the plane of grating $G_{2}$ is carried out by optical way, for the purpose, to eliminate various influences on image quality of moiré fringes. The grating $G_{2}$ is installed on single holder, who is held on rotary mount which can rotate about of optical axis for adjustment of the angle between the grating rulings. The grating $G_{2}$ is slightly rotated by optical axis by small angle $\Theta$ near zero degree; moiré pattern is formed on plane of grating $G_{2}$. The generation of moiré fringes system, by crossing of rulings gratings $\mathrm{G}_{1}$ and $\mathrm{G}_{2}$, consist of parallel bands alternately bright and dark, they are projected by the lenses system $\left(\mathrm{L}_{5}, \mathrm{~L}_{6}\right)$ on the observation plane OP, where the CCD camera is positioned and connected to the computer for automatic data processing.

\footnotetext{
a meguellati_said@yahoo.fr
} 


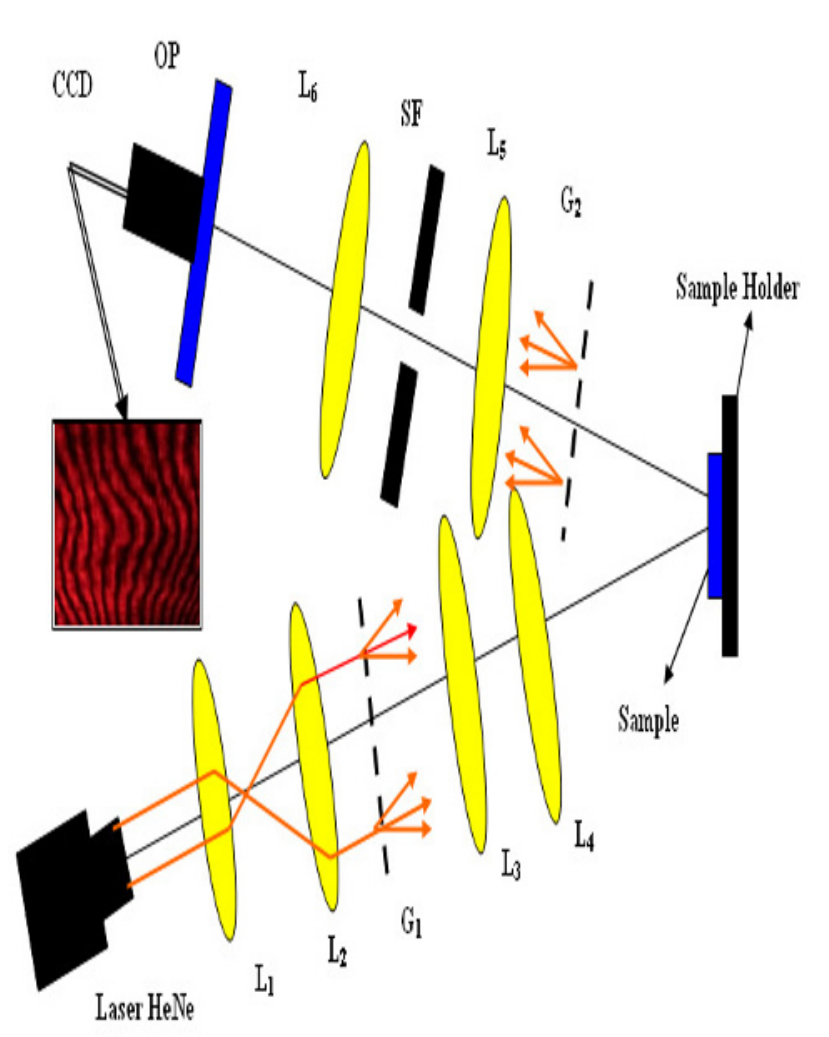

Figure 1. Optical principle of experimental setup.

\subsection{Scanning method of flatness defects}

The experimental setup shows in Figure 1., based on optical principle, is used to inspect the quality form of surface. First, the sample consists of a plane mirror with high flatness quality less $\lambda / 4$.The linear transmission grating $G_{1}$ is projected by the laser beam on the surface of mirror $M$ to be inspected. The grating image $G_{1}$ palpates the contours of the sample, will be printed of its topography, and is reflected to be superposed on the linear transmission grating $\mathrm{G}_{2}$ used as a reference grating. The light beam palpates the area of mirror $\mathrm{M}$; the structure of image grating $\mathrm{G}_{1}$ is impregnated of variations in the surface shape, and after reflection, is superimposed on the reference grating $G_{2}$. Modifications saved in the structure of grating image $G_{1}$ highlights defects of inspected surface form. The contours of moiré fringes change automatically and reproduce the defects form of surface of the mirror.

If the sample to be controlled presents a surface without defects, or at least defects undetectable by the sensibility control system, then the fringes consist of parallels straight lines alternatively bright and dark Figure 2 .

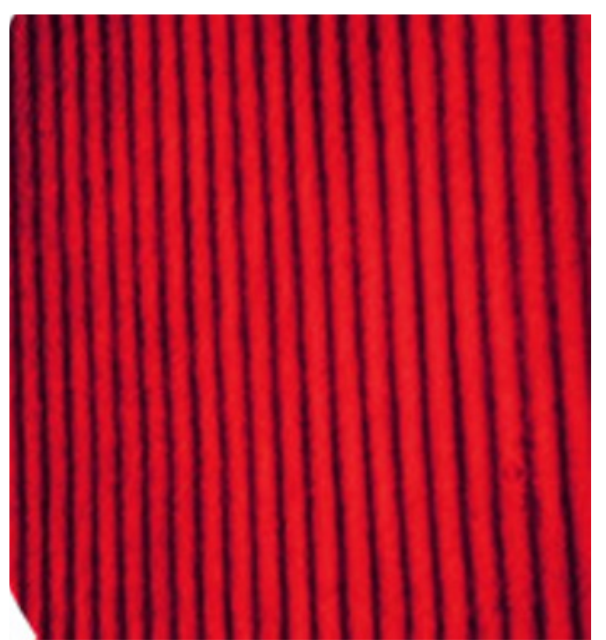

Figure 2. Moiré fringes of plan mirror flatness less $\lambda / 4$.

\section{Detection and resolution of measuring of flatness defects form}

\subsection{Detection of defects flatness form}

In the experimental setup of, Figure 1 and in the place of the mirror $\mathrm{M}$, we have used a stainless steel plate with reflection coefficient should be sufficient to allow observation of moiré fringes sufficient. If surface controlled contain defects, then while palpating surface, the image grating $G_{1}$ impregnates the exact shape of the controlled surface. The variation of the form of the structure of the image of grating $G_{1}$ and while being superimposed on the grating $G_{2}$ generates fringes of moiré which present variations in the contour of their forms, and the structure of moiré fringes are straight lines with slight fluctuations, which corresponds to small, thus materializing the localization of defects in shape of the surface object Figure 3.

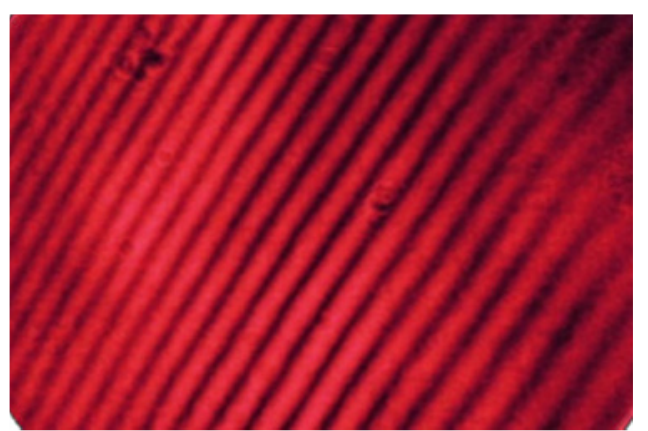

Figure 3. Moiré fringes of stainless steel plate without defects.

This fringe pattern means that the inspected surface has no defects, or sensitivity of the system is not sufficient to highlight defects form existing on this sample.

The defects forms existing on the surface inspected. In this case, the moiré fringes are not parallel straight lines, but have irregularities in their structures, which materialize the deformations on inspected surface of the sample. 
The significance or insignificance of defects form, materializing changes in the structure of moiré fringes, expressed the intensity of shape defects of the surface. Thus, any changes in the form of moiré fringes, provides direct information on the presence of defects form and its magnitude Figure 4.

Distortions or form defects, that a surface contains, are in fact displacements of dots on the surface. When these dots displacements are very weak, the difficulty of detection becomes important.

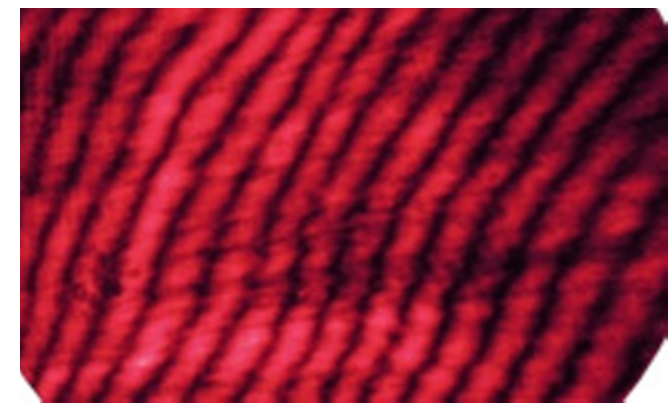

Figure 4. Moiré fringes of stainless steel plate with defects.

\subsection{Detection sensibility of experimental setup.}

This fringe pattern means that the inspected surface has no defects, or sensitivity of the system is not sufficient to highlight defects form existing on this sample.

The significance or insignificance of curvatures form, materializing changes in the structure of moiré fringes, expressed the intensity of shape defects of the surface. Thus, any changes in the form of moiré fringes, provides direct information on the presence of defects in shape and its magnitude.

Distortions or form defects, that a surface contains, are in fact displacements of dots on the surface. When these dots displacements are very weak, the difficulty of detection becomes important.

To highlight and observe the defects form with small magnitude, it is necessary to increase the sensitivity of moiré phenomenon by use of high spatial frequencies of grating and to call the fringe multiplication technique [10]. The setup according Fig. 1 contains the spatial filter SF which has the function to select diffraction orders (r, $0)$ and $(0, r)$ resulting from the superposition of the grating image $G_{1}$ and grating $G_{2}$. In complement, of the use, of the high spatial frequencies of gratings used, the selection of high harmonics $(r, 0)$ and $(0, r)$ of the diffraction pattern, increases the sensitivity of moiré fringes, which increases resolution of detection and thus allows, to make it possible to highlight microscopic defects form and lower.

The experimental setup described in this paper, is a precision assembly which allows the use of high spatial frequencies gratings and the use of spatial filtering technique or both them simultaneously, to increase sensitivity of moiré method, and consequently, increase resolution detection of control system. For detection and observation of deformations on a small surface by using this experimental setup, we have used a stainless steel plate with reflection coefficient should be sufficient to allow observation of moiré fringes. The sample is tested by the use of two linear transmission gratings of 100 lines $/ \mathrm{mm}$. With this spatial frequency, it is easy to measure defects on the order of $10^{-6} \mathrm{~m}$, and measurement uncertainty of $10^{-8} \mathrm{~m}$ without spatial filtering.

\section{Conclusions}

This measurement method is suitable for controlling distortions, defects of shape and flatness of precision surfaces, of optical and mechanical components, it also allows, measuring local defects on complex shapes, or objects goldsmithery.

The experimental setup, allows a considerable magnification of the image of inspected surface, it can detect defects at the microscopic level, through the magnification generated jointly by the moiré method which plays the role of movement microscope and the experimental setup itself., it is possible to measure easily micro defects on precision surface, with deformation of the micrometric range and uncertainty measurement of the order $10^{-8} \mathrm{~m}$. The sensitivity for displacement measurement using moiré technique depends on the frequency of the moiré grating, thus a great effort has been to get a grating with higher density.

This optical technique of non destructive control and contactless can be used advantageously to measure deformations generated by constraints on precision surface, and determining the functional influence, of these variations on the function. It can also be used for dimensional control, when, for example, to quantify the error in shape to whether an item is safe or rejected. Indeed, it then suffices to compare a figure of moiré fringes that is adapted to a given standard, so, that the system of moiré fringes is formed of equidistant parallel straight lines. Such moiré figure is previously recorded, which is very easy to use to test the conformance of the object to the standard, since it suffices to verify if the fringes contour is conform or not to the standard fringe contour, which saves precision, time and money.

\section{References}

1. H. Takazaki, Appl Opt Vol 12, Iss 4, 845-52, (1973)

2. H. Takazaki, S. Huzuoka, Opt Acta, GBR Vol 16 Iss $\mathrm{N}^{\circ} 8,1009-1019$ (1979)

3. SS. Ghorti, Opt and Lasers Eng, vol 48, Iss 2, 133140, ( 2010)

4. S. Zhang, Opt and lasers Eng, vol 48, Iss 2, 149$158,(2010)$

5. L. Chen, Y. Chang, Key Eng Mater, 113-6, (2008)

6. XU. Zhiguang, and Al, Opt Expr, Vol 17, Iss 21, 18394- 18407, ( 2009)

7. SS. Ghorti, G. Rajshekhar, P. Rastogi, Opt \& Las Tech, Vol 43, Iss 1, 40-44, (2011)

8. I. Admiror, The theory of moiré phenomenon (Kluwer Academic Publishers 2000)

9. M. Abdelhosani, Optik, 122, 510-513, (2011).

10. D. Post, Exp Mech USA, Vol 21, Iss N 3, 100-104, (1981) 